\title{
Knowledge-Driven Analytics and Systems Impacting Human Quality of Life
}

\author{
Arijit Ukil \\ TCS Research and \\ Innovation \\ Tata Consultancy Services \\ Kolkata, India \\ arijit.ukil@tcs.com.com
}

\author{
Leandro Marin \\ University of Murcia Spain \\ leandro@um.es
}

\author{
Antonio Jara \\ University of Applied \\ Sciences Western \\ Switzerland (HES-SO) \\ Switzerland \\ jara@ieee.org
}

\author{
John Farserotu \\ Centre Suisse \\ d'Electronique et de \\ Microtechnique (CSEM) \\ Switzerland \\ john.farserotu@csem.ch
}

\begin{abstract}
The advent of artificial intelligence (AI), Internet of Things (IoT), powerful computational hardwares like graphics processing units, affordable sensing devices like smart bands, wearables, smartphones pave ways for large number of useful and intelligent applications hitherto never commonly envisaged. However, it is felt that applications, which positively influence human life and society, need distinct attention from the perspective of the researchers, application developers as well as industry. It is understood that knowledge-driven initiatives in terms of technology, application and practical deployment have strong capability to enable long term human-centric convergence of cyber-physical systems. Our endeavor is to discuss those finer details, research directions and application development aspects of analytics and systems intended for impacting human quality of life.
\end{abstract}

\section{CCS Concepts}

- Computing methodologies Machine learning - Theory of computation $\sim$ Semi-supervised learning $\bullet$ Applied computing $\sim$ Health informatics $•$ Human-centered computing systems and tools

\section{Keywords}

Knowledge; Human Life; Artificial Intelligence; Sensors; Privacy; Big data; Deep Learning.

\section{MOTIVATION}

Knowledge-Driven Analytics and Systems Impacting Human Quality Of Life (KDAH), KDAH-CIKM-2019 is organized as a workshop in $28^{\text {th }}$ ACM International Conference on Information and Knowledge Management (CIKM), to be held during November 3rd-7th, 2019 in Beijing, China [1]. This workshop is in continuation of the previously organized KDAH-CIKM workshop in 27th ACM CIKM held during October, 2018 in Turin, Italy, [2][3].

Currently, we are at the critical moment of welcoming the

Permission to make digital or hard copies of part or all of this work for personal or classroom use is granted without fee provided that copies are not made or distributed for profit or commercial advantage and that copies bear this notice and the full citation on the first page. Copyrights for third-party components of this work must be honored. For all other uses, contact the Owner/Author.

CIKM '19, November 3-7, 2019, Beijing, China

(C) 2019 Copyright is held by the owner/author(s).

ACM ISBN 978-1-4503-6976-3/19/11.

https://doi.org/10.1145/3357384.3358799 influence of systems through knowledge-driven management analytics. The theme of CIKM 2019 "AI for Future Life" is appropriately aligned to the objective and goal of the workshop. AI has made significant impression to the research community and industry at large. KDAH-CIKM-2019 leverages those advancements and initiatives of $\mathrm{AI}$ and related enabler technologies to further expand the path of research and development towards human-centric application development and subsequent acceptance from the society.

Our main objective for organizing this workshop is to inculcate the research activities towards knowledge-driven human-centric applications. It is perceived that the rise of AI research associated with auxiliary technologies like IoT, ever-increasing computational power as well as the matured sensing technologies are holding the capability to significantly improve the human life and society. We notice that good amount research body is engaged in the advancement of machine intelligence algorithms and techniques. Convolution neural networks, generative adversarial networks, capsule networks are among many of the machine learning (aka deep learning) approaches to automate different tasks and augment the capability of machines to perform human duties. Such accomplishment of machine learning is getting possible to the continuous availability of knowledge in the forms of data-driven training of the machine learning algorithms. Apart from the availability of labelled data, other knowledge sources are also utilized to develop machine-learned applications including technologies for automated accomplishment of tasks by drones and robots. In [4], we have demonstrated that the data-driven management of cardiovascular screening can be reliably implemented as an important component of proactive, remote healthcare ecosystem. IoT based automated cardiac health screening is presented in [5] and it is understood that early detection of cardiac problem can be made possible by such types of systems.

However, the euphoria of the artificial intelligent systems has been marred with different types of undesirable implications and attacks. It is crucial to develop machine intelligence systems that can understand human language, expressions, contexts, actions, intentions and behaviors, and constantly interact with the sideeffects as well as voluntary and involuntary negative nuances. For example, a robotic surgery remotely performed if hacked, would likely to cause human death. Self-driven cars also require substantial protection against data security and hacking. Healthcare automation is one of the important applications of human-centric ecosystem development. Healthcare data is very much sensitive and privacy protection is of utmost importance. 
One of the prime requirements of developing sensor-enabled autonomous healthcare system for descriptive as well as prescriptive analytics is to deploy the client-side applications in embedded systems, which need a strict security enforcement of embedded device security [7]. Data privacy preservation such that different privacy breaching attacks can be nullified has been studied for years [8][9][10][11]. Privacy-preserving computation (e.g. homomorphic encryption [10]) has been considered as the holy grail of absolute prevention of data privacy attacks particularly when the computation or analytics job on the sensitive data is performed through a remote server or at the cloud. In order to minimize the security of private data for over air or other security-breaching attacks, we need to establish secure transmission channel and detect the different types of attacks [12][14]. In fact, human-centric applications deal with signals, data and information which are extracted from different sensors that closer to human beings. Sensors capture different human-related signals from wearable devices, smartphones, smart homes, smart vehicles to fetch the required information from human-beings and surrounding. Sensors form wireless sensor networks to exchange and amplify knowledge. Wireless sensor networks with powerful sensing and learning technologies form the core part of developing human-centric applications [13].

Another important objective of this workshop is to bring more focus on the augmented intelligence for analytics-driven human decisions and choices. Human decision-making require spectrum of skills and knowledge-driven analytics have the capability to provide the appropriate the direction in the game of choices and decisions. This workshop brings forward those positive applications and technologies as well as the path towards transformation of intelligent systems that minimize the notional and explicit negative impacts.

With the proliferation of AI-based applications and researches, we find large number of researchers from academia, industry and start-ups are engaged in developing technologies for knowledge-driven intelligent systems and human-centric applications like prediction of medical condition from healthcare data, personalization of medical treatment, developing the intelligent physical, emotional and mental diagnosis systems, detecting incoherence in human decisions and actions, forecasting financial fraud or investment opportunities, algorithmic trading, financial decision, proactive identification and prevention of data privacy breach and many more. This workshop provides the appropriate platform for the researchers to exchange ideas, present their works for a step forwarding in the research of human-centric applications.

\section{CONCLUSION}

Human-centric applications are the centerpiece for the development of human society. AI-based systems with the right conglomeration of knowledge, deep learning algorithms, sensors and IoT have the capability to create an eco-system of positive impacting systems and models that augment the human competence to usher a better world. We hope that the discussions, interactions and research outcome of KDAH-CIKM2019 workshop undoubtedly identify the direction, approaches and techniques to fulfill our goal of a better and improved human society. This workshop promotes collaboration and discussion among scholars from the domains of machine learning, knowledge management and engineering, data science, bio-informatics, data privacy and data security, and related others.

\section{ACKNOWLEDGEMENT}

Leandro Marin is partially supported by Research Project TIN2017-86885-R from the Spanish Ministery of Economy, Industry and Competitivity and Feder (European Union).

Antonio J. Jara is funded by the European Union's Horizon 2020 research and innovation programme under grant agreement No 732679, ACTIVAGE project https://www.activageproject.eu/.

\section{REFERENCES}

[1] https://sites.google.com/view/kdah-cikm-2019

[2] https://sites.google.com/view/knowledge-driven-analytics/home

[3] Arijit Ukil, Leandro Marin, Antonio Jara and John Farserotu, "Knowledge-driven Analytics and Sensor Signal Processing in Human-centric Applications," Knowledge-Driven Analytics Impacting Human Quality Of Life (KDAH) workshop in 27th ACM International Conference on Information and Knowledge Management (CIKM), 2018.

[4] Arijit Ukil, Antonio J. Jara, and Leandro Marin, "Data-Driven Automated Cardiac Health Management with Robust Edge Analytics and De-Risking," Sensors, June, 2019.

[5] Arijit Ukil, Uttam Kumar Roy. "Smart cardiac health management in IoT through heart sound signal analytics and robust noise filtering." IEEE 28th Annual International Symposium on Personal, Indoor, and Mobile Radio Communications (PIMRC), (2017).

[6] Arijit Ukil, Jaydip Sen, Sripad Koilakonda. "Embedded security for Internet of Things." IEEE National Conference on Emerging Trends and Applications in Computer Science (NCETACS), (2011): 1- 6.

[7] L. Sweeney. "Achieving k-anonymity Privacy Protection Using Generalization and Sup-pression.” Int. F. of Unc. Fuzz. Know. Syst, (2002): 571 - 588 .

[8] A. Machanavajjhala, D. Kifer, J, Gehrke, and M. Venkitasubramanian. "l-diversity:Privacy beyond k-anonymity." ACM Trans. Knowl. Disc. Data, vol. 1, issue. 1 (2007).

[9] A. Ukil, J. Sen, and S. Ghosh. "An Efficient Distribution Sensitive Privacy for Real-time Applications.” Computer Science and Convergence, LNEE, vol. 114, (2012): 81-91.

[10] Craig Gentry. "Fully Homomorphic Encryption Using Ideal Lattices.” ACM STOC, (2009): 169- 178.

[11] Arijit Ukil, Jaydip Sen. "Secure multiparty privacy preserving data aggregation by modular arithmetic." IEEE International Conference on Parallel Distributed and Grid Computing (PDGC), (2010): 344-349.

[12] J. Sen, S. Koilakonda, A. Ukil, "A mechanism for detection of cooperative black hole attack in mobile ad hoc networks," IEEE International Conference on Intelligent Systems, Modelling and Simulation (ISMS), pp. 338-343, 2011.

[13] Arijit Ukil, "Wireless Sensor Networks," In: Smart Wireless Sensor Networks, Intechopen, 2010.

[14] Arijit Ukil, Soma Bandyopadhyay, Abhijan Bhattacharyya, Arpan Pal, Tulika Bose, "Lightweight security scheme for IoT applications using CoAP," International Journal of Pervasive Computing and Communications, Volume 10, Issue 4, pp. 372-392, 2014. 